\title{
The Role of Primary Care in Improving Access to Medication-Assisted Treatment for Rural Medicaid Enrollees with Opioid Use Disorder
}

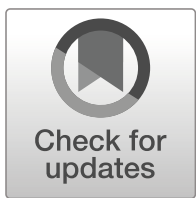

\author{
Evan S. Cole, $P h D^{7}$, Ellen DiDomenico, $M S^{2}$, Gerald Cochran, $P h D^{3}$, Adam J. Gordon, $M D^{3}$, \\ Walid F. Gellad, MD ${ }^{1,4}$, Janice Pringle, PhD ${ }^{5}$, Jack Warwick, MPH ${ }^{5}$, Chung-Chou H. Chang, PhD , \\ Joo Yeon Kim, MS ${ }^{7}$, Julie Kmiec, DO ${ }^{6}$, David Kelley, MD, MPA ${ }^{7}$, and Julie M. Donohue, $P h D^{7}$
}

\begin{abstract}
'Department of Health Policy and Management, University of Pittsburgh Graduate School of Public Health, Pittsburgh, PA, USA; ${ }^{\text {Pennsylvania }}$ Department of Drug and Alcohol Programs, Harrisburg, PA, USA; ${ }^{3}$ Department of Internal Medicine, Division of Epidemiology, University of Utah School of Medicine, Salt Lake City, UT, USA; 'Division of General Internal Medicine, University of Pittsburgh School of Medicine, Pittsburgh, PA, USA; ${ }^{5}$ Program Evaluation and Research Unit, University of Pittsburgh School of Pharmacy, Pittsburgh, PA, USA; ${ }^{6}$ Department of Psychiatry, University of Pittsburgh, Pittsburgh, PA, USA; ${ }^{7}$ Pennsylvania Department of Human Services, Harrisburg, PA, USA.
\end{abstract}

\begin{abstract}
BACKGROUND: The opioid epidemic has disproportionately affected rural areas, where a limited number of health care providers offer medication-assisted treatment (MAT), the mainstay of treatment for opioid use disorder (OUD). Rural residents with OUD may face multiple barriers to engagement in MAT including long travel distances.

OBJECTIVE: To examine the degree to which rural residents with OUD are engaged with primary care providers (PCPs), describe the role of rural PCPs in MAT delivery, and estimate the association between enrollee distance to MAT prescribers and MAT utilization.
\end{abstract}

DESIGN: Retrospective cohort study.

PARTICIPANTS: Medicaid-enrolled adults diagnosed with OUD in 23 rural Pennsylvania counties.

MAIN MEASURES: Primary care utilization, MAT utilization, distance to nearest possible MAT prescriber, mean distance traveled to actual MAT prescribers, and continuity of pharmacotherapy.

KEY RESULTS: Of the 7930 Medicaid enrollees with a diagnosis of OUD, a minority (18.6\%) received their diagnosis during a PCP visit even though enrollees with OUD had 4.1 visits to PCPs per person-year in 2015. Among enrollees with an OUD diagnosis recorded during a PCP visit, about half $(751,50.8 \%)$ received MAT, most of whom (508, 67.6\%) received MAT from a PCP. Enrollees with OUD with at least one PCP visit were more likely than those without a PCP visit to receive MAT (32.7\% vs. $25 \%$; $p<0.001$ ), and filled more buprenorphine and naltrexone prescriptions (mean $=11.1$ vs. 9.3; $p<0.001$ ). The median of the distances traveled to actual MAT prescribers was 48.8 miles, compared to a median of 4.2 miles to the nearest available MAT prescriber. Enrollees traveling a mean distance greater than 45 miles to MAT prescribers were less likely to receive continuity of pharmacotherapy (OR=0.71, 95\% CI = 0.56-0.91, $p=0.007)$.

$\overline{\text { Findings from this analysis were presented at the Addiction Health }}$ Services Research Conference on October 18, 2018.

Received November 1, 2018

Revised January 9, 2019

Accepted February 14, 2019

Published online March 18, 2019
CONCLUSIONS: PCP utilization among rural Medicaid enrollees diagnosed with OUD is high, presenting a potential intervention point to treat OUD, particularly if the enrollee's PCP is located nearer than their MAT prescriber.

KEY WORDS: primary care; medication-assisted treatment; rural; opioid use disorder.

J Gen Intern Med 34(6):936-43

DOI: $10.1007 / \mathrm{s} 11606-019-04943-6$

(c) Society of General Internal Medicine 2019

\section{INTRODUCTION}

Medication-assisted treatment (MAT) is the standard of care for opioid use disorder (OUD). ${ }^{1}$ Approved pharmacotherapies for OUD - buprenorphine, naltrexone, or methadone-differ substantially in the providers with authority to prescribe or dispense these medications. Naltrexone may be prescribed by any provider licensed to prescribe medications; buprenorphine may be prescribed only by providers who complete the DATA 2000 waiver training; methadone is only dispensed from specific licensed clinics. Under the current regulatory framework governing MAT providers, the emphasis of US policymakers has been on increasing access to office-based, mostly primary care, providers as opposed to clinics dispensing methadone. Indeed, the significant growth in supply of MAT providers in recent years was driven largely by physicians waivered to prescribe buprenorphine.

Unfortunately, improvements in MAT provider supply have not reached many rural areas; over $60 \%$ of small nonmetropolitan county residents still lived in an opioid treatment shortage area as of $2011 .^{2}$ Limits on the supply of MAT in rural areas is a critical public health problem given that rural areas in some states experienced nearly twofold higher increases in hospitalizations for opioid overdoses relative to urban areas in the last 15 years. ${ }^{3}$ Supply of MAT providers ${ }^{4}$ is limited in rural areas due to several factors, including provider attitudes towards addiction, ${ }^{5}$ a lack of provider 
training, ${ }^{6,7}$ and a limited supply of behavioral health providers. ${ }^{8,9}$ Furthermore, rural populations often travel long distances for primary and specialty health care, which can be associated with reduced utilization and poor health outcomes. ${ }^{10,11}$ The effect of distance to MAT providers on utilization and treatment outcomes is unknown. ${ }^{12}$

We seek to understand the potential for rural primary care providers (PCPs) to deliver MAT to patients with OUD by examining the following: (1) the degree to which rural residents with OUD are engaged in primary care, (2) rural PCPs current role in the delivery of MAT (buprenorphine and naltrexone), and (3) the association between distances from patients to MAT prescribers and utilization of MAT. We focus on Medicaid as our study setting as non-elderly adult Medicaid enrollees account for $12 \%$ of the adult population but $38 \%$ of those with an OUD. ${ }^{13}$

\section{METHODS}

\section{Overview}

We focused our analyses on adult Medicaid enrollees diagnosed with OUD, and within that group, enrollees with at least one primary care visit, and enrollees who received MAT. We conducted a cross-sectional comparison between enrollees with OUD who had $\geq 1$ primary care visit versus enrollees with OUD and no primary care visits. We conducted logistic regression analyses to estimate the association between distance to MAT prescribers and the likelihood that enrollees with OUD initiated MAT and received continuity of pharmacotherapy.

\section{Data}

We obtained Medicaid claims, encounter, and enrollment data for fee-for-service and managed care enrollees from 2014 to 2015 from the Pennsylvania Department of Human Services. Pennsylvania is the 7th largest Medicaid program by enrollment ${ }^{14}$ and the 4 th largest by expenditure ${ }^{15}$ and mirrors national averages on health care utilization although it has higher overdose death rates. Pennsylvania has the 3rd largest rural population. ${ }^{16}$ Pennsylvania expanded Medicaid under the Affordable Care Act in 2015, which some studies have shown to improve access to OUD treatment. ${ }^{17}$

\section{Patient Sample}

We included all full-benefit Medicaid-enrolled adults (age 1864) who were not dually eligible for Medicare. We limited our sample to residents of 23 rural counties with OUD prevalence and/or opioid/heroin overdose rates above the national average. Enrollees were included if they had $>1$ claim with a diagnosis of OUD (international classifications of diseases (ICD) version 9 and 10; see Appendix Table 4) in any diagnosis field during 2015. Diagnosis codes for OUD tend to have low sensitivity but high specificity, ${ }^{18,19}$ so we may underestimate the prevalence.

\section{MAT Utilization and MAT Setting}

As our focus was on MAT delivered by PCPs, our primary definition of MAT included buprenorphine and injectable or oral naltrexone but not methadone. We counted the number of fills or injections for buprenorphine (specifically formulations indicated for the treatment of OUD) or naltrexone per enrollee. We did, however, construct a variable for inclusion in descriptive analyses that measured the use of methadone, a potential substitute for buprenorphine or naltrexone, provided by methadone clinics based on procedure code. We categorized enrollees diagnosed with OUD in any of five service settings based on claim-level information: PCP visit, emergency department (ED) visit, acute care hospitalization, behavioral health visit (if the visit was covered by the enrollee's behavioral health managed care organization), or other (if they did not fit into one of the previous categories).

\section{Primary Care Utilization}

We constructed three measures of contact between patients with OUD and primary care: the number of primary care visits per person-year; how frequently enrollees who were diagnosed with OUD during a PCP visit received any MAT, and how frequently these enrollees received MAT from a PCP. We also identified if the enrollee had any claims for behavioral health counseling using a set of procedure codes. See Appendix Tables 5 and 6.

\section{Key Dependent Variables}

In our multivariable analyses, we had two key outcomes of interest: the likelihood that enrollees with OUD had any use of MAT (either buprenorphine or naltrexone) and the continuity of pharmacotherapy, which was defined using National Quality Forum specifications. Specifically, we identified enrollees with OUD initiating treatment with buprenorphine, naltrexone, and methadone who received $\geq 180$ days of continuous pharmacotherapy with no more than a 7 -day gap. ${ }^{20}$

\section{Key Independent Variable—Distance to MAT Provider}

To understand rural enrollees' access to MAT, we measured two distances: That between enrollees with OUD and the nearest possible MAT prescriber, and the distance traveled by enrollees to their actual MAT prescribers. For nearest possible MAT prescribers to enrollees in our study sample, we included prescribers with $\geq 1$ Medicaid-paid prescription fill for buprenorphine or naltrexone. We then identified the prescriber nearest to the center of each zip code, the geographic identifier available to us for enrollees, and measured the driving distance to the provider's office address using ArcGIS. We also examined distance to the nearest prescriber of 
buprenorphine or naltrexone to at least 10 Medicaid enrollees as some providers may prescribe to very few patients. To measure distance traveled to actual prescribers, we measured driving distance from the center of the enrollee's zip code to the prescribing provider's office address per prescription to account for prescriptions from different providers and providers that practice in multiple locations. We then took the mean of the distances traveled for the given enrollee. To be included in this measure, enrollees needed complete prescriber information (both on the claim and a valid address in the provider file) and continuous Medicaid enrollment without any gaps in coverage. We calculated the distribution of each distance measure by minimum, maximum, median, interquartile range, and 90th percentile.

\section{Covariates}

We included demographic characteristics including age, gender, race, and type of Medicaid eligibility: disabled, newly eligible (i.e., eligible through Medicaid expansion), nondisabled, and other. We also included measures of relevant comorbidities including anxiety, mood disorders, and schizophrenia and other psychotic disorders. ${ }^{21}$ We grouped enrollees into one of three regions in Pennsylvania: Northeast, Northwest, and Southwest. We calculated the number of chronic conditions for each enrollee using a modified version of the Elixhauser Comorbidity Index which used both inpatient and outpatient claims to identify conditions. ${ }^{22}$ We included indicators for which type of provider prescribed the greatest number of days supply of MAT: a PCP or a non-PCP. We categorized enrollees with at least one fill of methadone into a separate category since these patients are likely systematically different and PCPs cannot prescribe methadone.

\section{Statistical Analysis}

To test for differences between enrollees with OUD and $\geq 1$ primary care visit and enrollees with OUD and no primary care utilization, we used $t$ tests and chi-square tests as appropriate. To understand the association between distance to MAT prescribers and MAT treatment patterns, we used two logistic regression models. First, we estimated the association between distance to the nearest MAT prescriber on the likelihood of enrollees with OUD receiving MAT, controlling for the covariates listed above (except PCP or non-PCP MAT prescriber type). In this model, distance was specified as a continuous variable in miles, and we only included providers that prescribed to 10 or more enrollees, as including all prescribers may have underestimated distances to an active prescriber. Second, we estimated the association between the mean distance traveled to actual MAT prescribers and the likelihood of continuity of pharmacotherapy. We tested the mean distance traveled as both a continuous and a categorical variable. As a categorical variable, we created two groups based on mean distances traveled greater or less than 45 miles, as Pennsylvania regulations for Medicaid managed care organizations require that $90 \%$ of enrollees outside of metropolitan areas be located within 45 miles of primary and specialty care providers. ${ }^{23}$ In this model, we included the same covariates used in the model described above as well as PCP vs. non-PCP prescriber type.

We conducted two sensitivity analyses. First, we restricted the sample to those enrollees whose mean distance traveled was less than 270 miles to determine the influence of outliers on our estimates. The odds ratios and statistical significance were comparable to the main model. Second, we specified the mean distance traveled as a continuous variable in the model. The direction of the association and statistical significance remained the same as the categorical specification. We present the latter for ease of interpretation and for policy relevance given the 45-mile managed care regulation.

All analyses were conducted using SAS version 9.4. This study was approved under an expedited review by the University of Pittsburgh Institutional Review Board.

\section{RESULTS}

We identified 7930 Medicaid-enrolled adults who resided in the 23 rural counties and were diagnosed with an OUD in 2015 (Table 1). Among enrollees with OUD, 2361 (29.8\%) received MAT in 2015. Just over 55\% were enrolled through Medicaid expansion. Behavioral health diagnoses were highly prevalent: $42.5 \%$ were diagnosed with an anxiety disorder, $48.6 \%$ with a mood disorder, and $6.2 \%$ with schizophrenia or other psychotic disorder. Enrollees with OUD had 4.1 primary care visits per person-year.

Approximately $62 \%$ of rural enrollees with OUD had at least 1 primary care visit. Enrollees with a primary care visit were more likely to be older (mean age $=34.9$ vs. 30.9 ; $p<0.001)$ and female $(58.7 \%$ vs. $41 \% ; p<0.001)$ compared to those without a primary care visit. Over two-thirds of enrollees without primary care utilization were eligible through Medicaid expansion, compared to $47.7 \%$ of enrollees with a primary care visit. MAT utilization was higher among enrollees with primary care use, both in terms of the proportion receiving any MAT $(32.7 \%$ vs. $25 \% ; p<0.001)$, and in the mean number of buprenorphine or naltrexone fills/ injections (11.1 vs. 9.3; $p<0.001)$. Enrollees with primary care use were slightly less likely than those without to have any behavioral health counseling (37\% vs. $42.5 \%$; $p<0.001)$. Among enrollees with a primary care visit, 1478 enrollees (30.1\%) had their OUD diagnosis recorded on a PCP visit claim. Of that group, about half $(751,50.8 \%)$ received MAT. Most of these enrollees $(508,67.6 \%)$ received MAT from a PCP.

\section{Distance and MAT Utilization Analysis}

The median distance to the nearest possible MAT prescriber was 4.2 miles among rural enrollees with OUD (Fig. 1). The 90th percentile distance was 13.3 miles, and the maximum 
Table 1 Comparison of Enrollees with OUD in Sample Rural Counties Based on Primary Care Utilization, 2015

\begin{tabular}{|c|c|c|c|c|}
\hline & \multicolumn{3}{|c|}{ Enrollees with an OUD Diagnosis } & \multirow[b]{2}{*}{$p$} \\
\hline & All & $\begin{array}{l}\text { Enrollees who did } \\
\text { not have a primary } \\
\text { care visit }\end{array}$ & $\begin{array}{l}\text { Enrollees who had } \\
\geq 1 \text { primary care visit }\end{array}$ & \\
\hline Number of enrollees, $N(\%)$ & 7930 & $3015(38)$ & $4915(62)$ & \\
\hline Mean age, SD & $33.4(9.8)$ & $30.9(8.1)$ & $34.9(10.4)$ & $<0.001$ \\
\hline $18-29(\%)$ & $3561(44.9)$ & $1625(53.9)$ & $1936(39.4)$ & $<0.001$ \\
\hline $30-39(\%)$ & $2669(33.7)$ & $1028(34.1)$ & $1641(33.4)$ & \\
\hline $40-49(\%)$ & $1004(12.7)$ & $247(8.2)$ & $757(15.4)$ & \\
\hline $50-64(\%)$ & $696(8.8)$ & $115(3.8)$ & $581(11.8)$ & \\
\hline Female, $N(\%)$ & $4123(52)$ & $1236(41)$ & $2887(58.7)$ & $<0.001$ \\
\hline \multicolumn{5}{|l|}{ Eligibility type, $N(\%)$} \\
\hline Disabled & $1562(19.7)$ & $341(11.3)$ & $1221(24.8)$ & $<0.001$ \\
\hline Newly eligible & $4383(55.3)$ & $2039(67.6)$ & $2344(47.7)$ & \\
\hline Non-disabled adults & $1652(20.8)$ & $578(19.2)$ & $1074(21.9)$ & \\
\hline Other & $333(4.2)$ & $57(1.9)$ & $276(5.6)$ & \\
\hline \multicolumn{5}{|l|}{ Race, $N(\%)$} \\
\hline Non-Hispanic White & $7620(96.1)$ & $2887(95.8)$ & $4733(96.3)$ & 0.562 \\
\hline Non-Hispanic Black & $174(2.2)$ & $74(2.5)$ & $100(2)$ & \\
\hline Hispanic & $74(0.9)$ & $31(1)$ & $43(0.9)$ & \\
\hline Other & $62(0.8)$ & $23(0.8)$ & $39(0.8)$ & \\
\hline \multicolumn{5}{|l|}{ Enrollees with a behavioral health diagnosis, $N(\%)$} \\
\hline Anxiety disorder & $3369(42.5)$ & $715(23.7)$ & $2654(54)$ & $<0.001$ \\
\hline Mood disorder & $3850(48.6)$ & $937(31.1)$ & $2913(59.3)$ & $<0.001$ \\
\hline Schizophrenic and other psychotic disorders & $488(6.2)$ & $127(4.2)$ & $361(7.3)$ & $<0.001$ \\
\hline Enrollees who received MAT, $N(\%)$ & $2361(29.8)$ & $754(25)$ & $1607(32.7)$ & $<0.001$ \\
\hline Number of MAT fills, mean (SD) & $10.6(8.3)$ & $9.3(7.7)$ & $11.1(8.5)$ & $<0.001$ \\
\hline Enrollees with a claim for counseling, $N(\%)$ & $3114(39.3)$ & $1281(42.5)$ & $1833(37.3)$ & $<0.001$ \\
\hline Number of primary care Visits per person-year & 4.1 & 0 & 6.2 & \\
\hline Number of primary care visits, mean (SD) & $3.5(5.2)$ & $0(0)$ & $5.6(5.7)$ & \\
\hline OUD diagnosed during PCP visit, $N(\%)$ & $1478(18.6)$ & N/A & $1478(30.1)$ & \\
\hline Enrollees who received MAT among those & $751(50.8)$ & N/A & $751(50.8)$ & \\
\hline \multicolumn{5}{|l|}{ diagnosed during PCP visit, $N(\%)$} \\
\hline Enrollees who received MAT from a PCP, & $508(34.4)$ & $\mathrm{N} / \mathrm{A}$ & $508(34.4)$ & \\
\hline among those diagnosed during PCP visit, $N(\%)$ & & & & \\
\hline \multicolumn{5}{|c|}{ Diagnosed with OUD in other places of service, $N(\%)$} \\
\hline ED Visit & $784(9.9)$ & $283(9.4)$ & $501(10.2)$ & 0.243 \\
\hline Acute care hospitalization & $589(7.4)$ & $182(6)$ & $407(8.3)$ & $<0.001$ \\
\hline Behavioral health visit & $5684(71.7)$ & $2474(82.1)$ & $3210(65.3)$ & $<0.001$ \\
\hline Other & $3688(46.5)$ & 1198 (39.7) & $2490(50.7)$ & $<0.001$ \\
\hline
\end{tabular}

MAT includes buprenorphine, injectable naltrexone, and oral naltrexone

was 44.1 miles and, thus, within the 45-mile managed care requirement. When restricted to providers who prescribed MAT to at least 10 enrollees, the median increased to 12.6 miles, the 90th percentile to 25.4 miles, and the maximum to 65.8 miles. We identified 1186 enrollees $(50.2 \%)$ with OUD receiving MAT who met our criteria to be included in the distance traveled to the actual MAT prescriber measure. Among this population, the mean distance traveled to actual prescribers ranged from 0.8 to 395.9 miles, with a median of 48.8 miles, and an interquartile range of 23.3 to 87.8 miles. Approximately 88\% (1049 enrollees) received a prescription for MAT from a provider who prescribed to 10 or more patients.

The distance to the nearest MAT prescriber was significantly associated with the likelihood of receiving any MAT (OR = $0.988 ; 95 \% \mathrm{CI}=0.983-0.993 ; p<0.001)$, indicating that every additional mile was associated with a $1.2 \%$ reduction in the odds of receiving any MAT (Table 2).

Among enrollees with OUD who received MAT and had complete prescriber information $(n=1186), 580(48.9 \%)$ received continuity of pharmacotherapy for at least 180 days.
We found a number of variables were important in maintaining pharmacotherapy continuity (Table 3). Enrollees who traveled a mean distance of 45 miles or more had approximately $29 \%$ lower odds in receiving continuity of pharmacotherapy for OUD relative to those who traveled less than 45 miles (OR = $0.71 ; 95 \% \mathrm{CI}=0.56-0.91 ; p=0.007)$. In addition, enrollees with a diagnosis of schizophrenia or other psychotic disorder were significantly less likely to have continuity of pharmacotherapy $(\mathrm{OR}=0.43 ; 95 \% \mathrm{CI}=0.23-0.82 ; p=0.01)$. Each additional comorbidity from the Elixhauser Index was associated with a decrease of $10 \%(\mathrm{OR}=0.90 ; 95 \% \mathrm{CI}=0.82-0.98 ; p=$ 0.016 ) in the odds of continuity of pharmacotherapy. Finally, enrollees who received the majority of their MAT from a nonPCP provider were more likely to have continuity of pharmacotherapy than enrollees who received the majority of their MAT from a PCP $(\mathrm{OR}=1.33 ; 95 \% \mathrm{CI}=1.0-1.7 ; p=0.04)$. Enrollees with any methadone fill were substantially less likely to have continuity of pharmacotherapy relative to enrollees who received their MAT from a $\mathrm{PCP}(\mathrm{OR}=0.27$; $95 \% \mathrm{CI}=0.16-0.45 ; p<0.001)$. 
180

90th Pctl

160

140

60

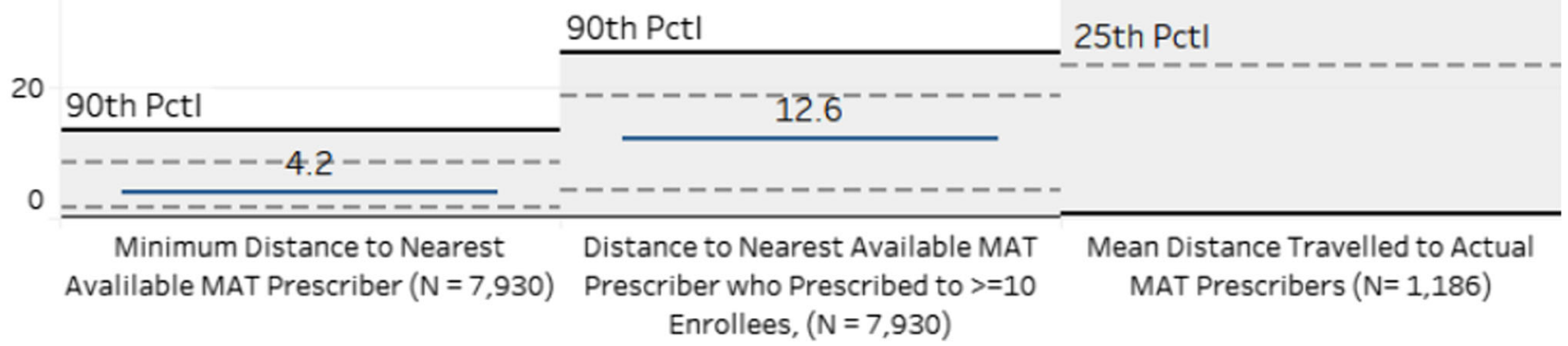

Fig. 1 Distribution of Distances to Nearest MAT Prescriber and Mean Distance Traveled to MAT Prescribers, in Miles (2014-2015). MAT prescriber includes the service location of a provider who prescribed buprenorphine, injectable naltrexone, or oral naltrexone to any Medicaid enrollee in 2015. Distance was taken from the center of the patient's zip code to the service location address of the provider, and measured as a driving distance. Mean distance traveled to MAT prescriber was calculated across all of 2014 and 2015 for adult enrollees who were eligible for

the NQF Continuity of Pharmacotherapy measure. To be included, enrollees had to (1) be diagnosed with OUD; (2) have $\geq 1$ claim for buprenorphine, injectable naltrexone, or oral naltrexone; (3) be continuously enrolled for at least 6 months; (4) have no missing prescriber IDs for MAT in the pharmacy file; and (5) have valid service location addresses for each MAT prescriber. Interquartile, median, and 90th percentile presented. 90th percentile presented instead of maximum for presentation purposes. Maximum values were the following: minimum distance to nearest available MAT prescriber $=44.1$ miles; distance to nearest available MAT prescriber who prescribed to $\geq 10$ enrollees $=65.8$ miles; mean distance traveled to actual MAT prescribers $=395.9$ miles.

\section{DISCUSSION}

We found that Medicaid-enrolled adults with OUD in rural areas use primary care frequently, but that less than $20 \%$ are diagnosed with their OUD during a PCP visit. The proportion of enrollees with a buprenorphine or naltrexone fill/injection and the mean number of fills/injections were higher for enrollees who had at least one PCP visit compared to enrollees with no primary care visits. Rural Medicaid enrollees with OUD traveled distances nearly 4 times longer to their actual
MAT prescribers than the median distance of the MAT prescriber nearest to all enrollees with OUD, and that greater distances to MAT prescriber were associated with lower odds of receiving any MAT and receiving continuity of pharmacotherapy for OUD.

An important finding from this analysis is that rural Medicaid enrollees with OUD have substantial contact with their PCPs, with over 4 visits per person-year. This demonstrates that PCPs are well-positioned to treat patients with OUD. PCPs may be reluctant to provide MAT services in part due 
Table 2 Logistic Regression on the Likelihood of Receiving MAT, $2015(N=7930)$

\begin{tabular}{|c|c|c|}
\hline & Odds ratio $(95 \% \mathrm{CI})$ & $p$ \\
\hline Distance to nearest MAT provider & $0.988(0.983-0.993)$ & $<0.001$ \\
\hline \multicolumn{3}{|l|}{ Age $(\mathrm{ref}=50-64)$} \\
\hline $\begin{array}{l}18-29 \\
30-39\end{array}$ & $\begin{array}{l}2.24(1.75-2.87) \\
2.49(1.94-3.19)\end{array}$ & $\begin{array}{l}<0.001 \\
<0.001\end{array}$ \\
\hline $40-49$ & $1.75(1.33-2.28)$ & $<0.001$ \\
\hline \multicolumn{3}{|l|}{ Race (ref = White) } \\
\hline Non-White & $0.58(0.43-0.78)$ & $<0.001$ \\
\hline $\begin{array}{l}\text { Gender }(\text { ref = male }) \\
\text { Female }\end{array}$ & $1.22(1.10-1.35)$ & $<0.001$ \\
\hline \multicolumn{3}{|l|}{ Eligibility (ref = non-disabled) } \\
\hline Disabled & $0.76(0.64-0.89)$ & 0.001 \\
\hline Newly eligible & $0.65(0.57-0.74)$ & $<0.001$ \\
\hline Other & $0.73(0.55-0.97)$ & 0.028 \\
\hline \multicolumn{3}{|l|}{ MCO region (ref = Northwest) } \\
\hline Northeast & $1.03(0.92-1.15)$ & 0.623 \\
\hline Southwest & $0.87(0.76-1.00)$ & 0.052 \\
\hline Anxiety disorder & $1.27(1.14-1.43)$ & $<0.001$ \\
\hline Mood disorder & $1.12(0.99-1.27)$ & 0.067 \\
\hline $\begin{array}{l}\text { Schizophrenia and other } \\
\text { psychotic disorder }\end{array}$ & $0.76(0.60-0.97)$ & 0.025 \\
\hline Elixhauser Index & $0.90(0.87-0.93)$ & $<0.001$ \\
\hline
\end{tabular}

2361 enrollees (29.8\%) received MAT. Nearest MAT provider was restricted to providers that prescribed buprenorphine or naltrexone to at least 10 enrollees during 2015. In addition, among prescribers that only prescribed naltrexone, we restricted to prescribers who prescribed to at least one enrollee diagnosed with OUD, as naltrexone can be prescribed for alcohol use disorder as well

to stigma towards patients with addiction. ${ }^{5}$ By showing PCPs that they already serve patients with OUD, and that by adopting MAT they would not need to expand patient volume, payers and policymakers may encourage MAT delivery by PCPs to patients they routinely serve. We also found that the majority of enrollees with at least one primary care visit were never diagnosed with their OUD in a primary care setting, which may be due to suboptimal screening for OUD in primary care. However, it is of interest that over half of enrollees diagnosed with OUD during a PCP visit received MAT, and often from a PCP versus only $29.8 \%$ of all rural enrollees with OUD. This could indicate that when PCPs identify OUD, they play a crucial role in MAT initiation.

The relationship between travel distance and MAT treatment points to the importance of referring patients to more conveniently located MAT providers whenever possible. In addition, if the enrollee's PCP is closer than their MAT prescriber, this demonstrates another potential benefit to delivering MAT within primary care; however, we did find that enrollees who received the majority of their MAT from a PCP were less likely to achieve continuity of pharmacotherapy than those who received the majority of their MAT from a nonPCP. This association could be due to differences in the quality of care or to differences in the patient populations that we were not able to control for in this observational study.

Our analysis is subject to a few important limitations. First, like all claims-based analyses, our results are influenced by how providers bill for their services and which diagnoses they code. Second, we based our definition of PCPs on provider type and specialty codes which may have led to misclassifications of certain providers. We took an additional step of
Table 3 Logistic Regression on the Likelihood of Receiving Continuity of Pharmacotherapy, 2014-2015 $(N=1186)$

\begin{tabular}{|c|c|c|}
\hline & $\begin{array}{l}\text { Odds ratio } \\
(95 \% \text { CI })\end{array}$ & $p$ \\
\hline \multicolumn{3}{|l|}{ Mean distance traveled (ref $\leq 45$ miles) } \\
\hline \multicolumn{3}{|l|}{ Age $(\mathrm{ref}=50-64)$} \\
\hline $18-29$ & $0.86(0.47-1.56)$ & 0.614 \\
\hline $30-39$ & $1.07(0.59-1.94)$ & 0.822 \\
\hline $40-49$ & $1.02(0.53-1.97)$ & 0.961 \\
\hline \multicolumn{3}{|l|}{ Race (ref= White) } \\
\hline \multirow{2}{*}{\multicolumn{3}{|c|}{ Gender $($ ref = male $)$}} \\
\hline & & \\
\hline Female & $1.39(1.06-1.81)$ & 0.016 \\
\hline \multicolumn{3}{|l|}{ Eligibility (ref = non-disabled) } \\
\hline Disabled & $1.03(0.77-1.38)$ & 0.849 \\
\hline Newly eligible & $0.34(0.22-0.53)$ & $<0.001$ \\
\hline Other & $1.13(0.65-1.98)$ & 0.657 \\
\hline \multicolumn{3}{|l|}{ MCO region (ref = Northwest) } \\
\hline Northeast & $0.59(0.44-0.78)$ & $<0.001$ \\
\hline Southwest & $0.88(0.62-1.24)$ & 0.468 \\
\hline Anxiety disorder & $1.01(0.77-1.32)$ & 0.950 \\
\hline Mood disorder & $1.03(0.76-1.41)$ & 0.831 \\
\hline $\begin{array}{l}\text { Schizophrenia and other psychotic } \\
\text { disorder }\end{array}$ & $0.43(0.23-0.82)$ & 0.010 \\
\hline Elixhauser Index & $0.90(0.82-0.98)$ & 0.016 \\
\hline \multicolumn{3}{|c|}{$\begin{array}{l}\text { Majority of MAT days (ref = majority of } \\
\text { buprenorphine/naltrexone prescribed by PCP) }\end{array}$} \\
\hline Any days on methadone & $0.27(0.16-0.45)$ & $<0.001$ \\
\hline $\begin{array}{l}\text { Majority of buprenorphine/naltrexone } \\
\text { prescribed by non-PCP }\end{array}$ & $1.33(1.01-1.74)$ & 0.040 \\
\hline
\end{tabular}

580 enrollees (48.9\%) met the criteria for continuity of pharmacotherapy. By majority of MAT days group, this figure was $49.5 \%$ for PCPS $(n=366) ; 52.9 \%$ for non-PCPs $(n=709)$; and $21.6 \%$ for those with any methadone fill $(n=111)$. Mean distance traveled to MAT prescribers was calculated across all of 2014 and 2015 for adult enrollees who were eligible for the NQF Continuity of Pharmacotherapy. To be included, enrollees had to (1) be diagnosed with OUD; (2) have $\geq 1$ claim for buprenorphine, injectable naltrexone, or oral naltrexone; (3) be continuously enrolled for at least 6 months; (4) have no missing prescriber IDs for MAT in the pharmacy file, and (5) have valid service location addresses for each MAT prescriber

examining subspecialties listed in NPPES to address this issue, and thus believe it is a minor concern. Third, without access to enrollee address, we mapped the center of the enrollee's zip code to provider address to measure distance thus introducing some measurement error in provider distances. In addition, some distances traveled at the higher end of the distribution could be due to enrollment or claims data errors; however, our results were consistent in the sensitivity analysis when these distances were excluded. Finally, while we controlled for demographic and health-related conditions in our logistic regression models, our models may be subject to unmeasured confounding related to enrollee transportation access and health status.

Our analysis shows that while Medicaid enrollees in rural areas face challenges to obtaining MAT services, there is an opportunity to expand access to MAT through primary care. Medicaid-enrolled adults with OUD are already using primary care services, serving as an intervention point for the health care system. Policymakers should consider efforts that encourage MAT adoption among PCPs to expand access to opioid addiction treatment in rural areas. Furthermore, given our findings on the association between distance and MAT 
utilization, policymakers should focus on rural areas where Medicaid enrollees are traveling long distances to their MAT prescriber. Future research should explore the reasons behind rural Medicaid enrollee travel patterns for MAT, and why they might bypass prescribers that are substantially closer.

Corresponding Author: Evan S. Cole, PhD; Department of Health Policy and Management, University of Pittsburgh Graduate School of Public Health, Pittsburgh, PA, USA (e-mail: evancole@pitt.edu).

Funding Information This study was funded by a grant from the Agency for Healthcare Research \& Quality (1R18HSO25072-01).

\section{Compliance with Ethical Standards:}

Conflict of Interest: The authors declare that they do not have a conflict of interest.

\section{REFERENCES}

1. Substance Abuse and Mental Health Services Administration. Medication-Assisted Treatment. 2017; https://www.samhsa.gov/medication-assisted-treatment. Accessed 13 Aug 2017.

2. Dick AW, Pacula RL, Gordon AJ, et al. Growth In Buprenorphine Waivers For Physicians Increased Potential Access To Opioid Agonist Treatment, 2002-11. Health Aff. 2015;34(6):1028-1034.

3. PA Health Care Cost Containment Council. Hospitalizations for Overdose of Pain Medication and Heroin. Harrisburg, PA; 2016.

4. Rosenblatt RA, Andrilla CH, Catlin M, Larson EH. Geographic and specialty distribution of US physicians trained to treat opioid use disorder. Ann Fam Med. 2015;13(1):23-26.

5. Substance Abuse and Mental Health Services Administration. 2014 Buprenorphine Summit. Rockville, MD; 2014.

6. Barry DT, Irwin KS, Jones ES, et al. Integrating buprenorphine treatment into office-based practice: a qualitative study. J Gen Intern Med. 2009;24(2):218-225.

7. Oliva EM, Maisel NC, Gordon AJ, Harris AH. Barriers to use of pharmacotherapy for addiction disorders and how to overcome them. Curr Psychiatry Rep. 2011;13(5):374.

8. Hutchinson E, Catlin M, Andrilla CHA, Baldwin L-M, Rosenblatt RA. Barriers to primary care physicians prescribing buprenorphine. Ann Fam Med. 2014; 12(2): 128-133.

9. Kissin W, McLeod C, Sonnefeld J, Stanton A. Experiences of a national sample of qualified addiction specialists who have and have not prescribed buprenorphine for opioid dependence. J Addict Dis. 2006;25(4):91-103.

10. Buzza C, Ono SS, Turvey C, et al. Distance is relative: unpacking a principal barrier in rural healthcare. J Gen Intern Med. 2011;26 Suppl 2:648-654.

11. Kelly C, Hulme C, Farragher T, Clarke G. Are differences in travel time or distance to healthcare for adults in global north countries associated with an impact on health outcomes? A systematic review. BMJ Open. 2016;6(11):e013059.

12. Luu H, Slavova S, Freeman PR, Lofwall M, Browning S, Trends $\mathbf{H}$. Trends and Patterns of Opioid Analgesic Prescribing: Regional and RuralUrban Variations in Kentucky From 2012 to 2015. J Rural Health. 2018.

13. Zur J, Tolbert $\mathbf{J}$. The Opioid Epidemic and Medicaid's Role in Facilitating Access to Treatment. 2018. https://www.kff.org/medicaid/issue-brief/ the-opioid-epidemic-and-medicaids-role-in-facilitating-access-to-treatment/. Accessed 16 May 2018.

14. Centers for Medicare and Medicaid Services. Medicaid Enrollment Data Collected Through MBES. 2018. https://www.medicaid.gov/medicaid/ program-information/medicaid-and-chip-enrollment-data/enrollmentmbes/index.html. Accessed 17 Sept 2018.

15. Kaiser Family Foundation. Total Medicaid Spending, FY 2016. 2018. https://www.kff.org/medicaid/state-indicator/total-medicaid-spending / ? currentTimeframe $=0$ \& sort Model = \% 7 B $\% 22$ colld
$\% 22: \% 22$ Total $\% 20$ Medicaid $\% 20$ Spending\%22,\%22 sort $\% 22: \% 22$ desc\%22\%7D. Accessed 17 Sept 2018.

16. United States Census Bureau. Lists of Population, Land Area, and Percent Urban and Rural in 2010 and Changes from 2000 to 2010. 2010. https://www.census.gov/geo/reference/ua/urban-rural-2010. html. Accessed 19 Jan 2018.

17. McKenna RM. Treatment use, sources of payment, and financial barriers to treatment among individuals with opioid use disorder following the national implementation of the ACA. Drug Alcohol Depend. 2017;179:8792.

18. Kim HM, Smith EG, Stano CM, et al. Validation of key behaviourally based mental health diagnoses in administrative data: suicide attempt, alcohol abuse, illicit drug abuse and tobacco use. BMC Health Serv Res. 2012;12(1): 18 .

19. Rowe C, Vittinghoff E, Santos GM, Behar E, Turner C, Coffin PO. Performance Measures of Diagnostic Codes for Detecting Opioid Overdose in the Emergency Department. Acad Emerg Med Off $J$ Soc Acad Emerg Med. 2017;24(4):475-483.

20. National Quality Forum. Behavioral Health 2016-2017. Washington DC; 2017.

21. Chronic Conditions Data Warehouse. Condition Categories. 2018. https://www.ccwdata.org/web/guest/condition-categories. Accessed 16 May 2018.

22. Elixhauser A, Steiner C, Harris DR, Coffey RM. Comorbidity measures for use with administrative data. Med Care. 1998;36(1):8-27.

23. Health Maintenance Organization Act, § 9.679 The Administrative Code of 1929. 2001.

\section{APPENDIX}

Table 4 Diagnosis Codes Used to Identify OUD

\begin{tabular}{ll}
\hline \hline Any ICD-9-CM that starts with & Any ICD-10-CM that starts with \\
\hline 3040 & F11 \\
3047 & \\
3055 & \\
\hline
\end{tabular}

\section{PCP Identification}

PCPs were identified based on provider specialty, place of service, and procedure code criteria. First, we classified providers as PCPs if they indicated a specialty of pediatrics, family health, nurse practitioner (in a primary care setting), physician assistant, family practice, general practitioner, internal medicine, or general internist in the Pennsylvania Medicaid provider file. To distinguish between internal medicine physicians with and without sub-specialty training we linked to National Plan and Provider Enumeration System (NPPES) data and classified physicians as PCPs if they only listed specialties included in the list above.

Next, we identified primary care-related visits to these providers if the claim had one of the following procedure code types: evaluation and management visits, consultations, physical exams, annual wellness visits, and federally qualified health center (FQHC) or rural health center (RHC) specific procedure codes, and office, outpatient hospital, FQHC, or RHC places of service. 
Table 5 Procedure Codes Used in Primary Care Visit Criteria

\begin{tabular}{ll}
\hline $\begin{array}{l}\text { For primary care performing } \\
\text { providers }\end{array}$ & $\begin{array}{l}\text { For FQHC/RHC performing } \\
\text { providers }\end{array}$ \\
\hline 99201-99205 & $\begin{array}{l}\text { T1015, with MOD1 not in } \\
\text { ("HE", "U9", "HF") }\end{array}$ \\
$99211-99215$ & \\
$99381-99387$ & \\
$99241-99245$ & \\
$99271-99275$ & \\
99499 & \\
G0402 & \\
G0438 & \\
\hline
\end{tabular}

Table 6 Procedure Codes Used to Identify Counseling Claims

\begin{tabular}{llllll}
\hline \hline H0049 & H0050 & H2034 & H2035 & H2036 & H0047 \\
H0014 & H0012 & H0013 & H0008 & H0007 & H0015 \\
H0004 & H0005 & H0028 & T1006 & H0001 & H0006 \\
\hline
\end{tabular}

Publisher's Note: Springer Nature remains neutral with regard to jurisdictional claims in published maps and institutional affiliations. 